'Departamento de Nutrición y Dietética y Programa de Magister en Nutrición Humana, Facultad de Farmacia, Universidad de Concepción. Concepción, Chile.

2BHF Glasgow Cardiovascular Research Centre, Institute of Cardiovascular and Medical Science, University of Glasgow, Glasgow, United Kingdom. ${ }^{3}$ Instituto de Farmacia, Facultad de Ciencias, Universidad Austral de Chile. Valdivia, Chile. ${ }^{4}$ Instituto de Anatomía, Histología y Patología, Facultad de Medicina, Universidad Austral de Chile. Valdivia, Chile.

${ }^{5}$ Escuela de Educación Física, Universidad San Sebastián. Concepción, Chile.

${ }^{6}$ Escuela de Kinesiología, Facultad de Salud Universidad Santo Tomás, Sede Valdivia. Chile.

${ }^{7}$ Grupo de Investigación Calidad de Vida Departamento de Ciencias de la Educación, Universidad del Biobío, Chillán, Chile.

${ }^{8}$ Departamento de Educación Física. Facultad

de Educación. Universidad de Concepción. Concepción, Chile.

${ }^{9}$ Centro de Fisiología y Biomecánica, Universidad Mayor, Santiago, Chile.

${ }^{a}$ Nutricionista. MSc. Nutrición Humana.

${ }^{b}$ Nutricionista. MSc. Planificación en Alimentación y Nutrición

Nutricionista. MSc. Ciencias de la Educación.

${ }^{d}$ Bioquímica. MSc. Nutrición y Dietética. eProfesora de Biología y Química. MSc Neurociencias y Salud Mental. fProfesor de Educación Física, Doctor en Actividad física, Educación Física y Deporte. 9Profesor de Educación Física, MSc. Educación en Salud y Bienestar Humano.

hProfesor de Educación Física, MSc Educación. iProfesor de Educación Física, MSc Educación Física.

jProfesor de Educación Física, Doctor en Ciencias Cardiovasculares y Biomédicas.

*FP, AML y ED contribuyeron igualmente y son consideradas primeras autoras compartidas.

Ninguno de los autores reporta tener conflictos de intereses.

Recibido el 6 de mayo de 2017, aceptado el 29 de agosto de 2017.

Correspondencia a: Dr. Carlos Celis-Morales

BHF Glasgow Cardiovascular Research Centre, 126 University Avenue, Glasgow University Glasgow, United Kingdom, G12 8TA carlos.celis@glasgow.ac.uk

\section{Factores de riesgo asociados al desarrollo de hipertensión arterial en Chile}

\author{
FANNY PETERMANN 1 1,2,a,*, ELIANA DURÁN $N^{1, b, *}$, \\ ANA MARÍA LABRAÑA ${ }^{1, \mathrm{c}, *}$, MARÍA ADELA MARTÍNEZ ${ }^{3, \mathrm{~d}}$, \\ ANA MARÍA LEIVA, ${ }^{4, e}$, ALEX GARRIDO-MÉNDEZ ${ }^{5, \mathrm{f}}$, \\ FELIPEPOBLETE-VALDERRAMA ${ }^{6, g}$, XIMENADÍAZ-MARTÍNEZ $^{7, \mathrm{~h}}$, \\ CARLOS SALAS ${ }^{8, \mathrm{i}}$, CARLOS CELIS-MORALES ${ }^{2,9, \mathrm{j}}$
}

\section{Risk factors associated with hypertension. Analysis of the 2009-2010 Chilean health survey}

Background: High blood pressure is one of the major risk factors for the development of cardiovascular disease, affecting 27\% of the Chilean population in 2010. Aim: To determine the risk factors associated with the development of hypertension. Material and Methods: Analysis of the database of the 2009-2010 National Health Survey in which 4,901 participants were included. Socio-demographic factors, physical activity, eating habits, well-being and comorbidities were analyzed. Results: Women had a lower risk of developing hypertension than men (Odds ratio (OR): 0.69, $95 \%$ confidence intervals $(C I): 0.59-0.81, p<0.01)$. In both men and women, the risk is greater over the age of 25 years (OR: 2.90, $95 \%$ CI: 1.55-5.43, $p<0.01)$. The risk is greater in subjects who were overweight (OR: 1.61, 95\% CI: 1.31-1.98, $p<0.01$ ), obese (OR: 3.18, 95\% CI: 2.56-3.95, $p<0.01$ ), or had central obesity (OR: 2.25, 95\% CI: 1.85-2.72, $p<0.01$ ). Subjects with a family history of hypertension and diabetes also have a higher risk. Conclusions: Identifying the risk factors associated with hypertension allows public health policies to be tailored to its prevention.

(Rev Med Chile 2017; 145: 996-1004)

Key words: Diet; Exercise; Hypertension; Lyfe Style; Obesity.

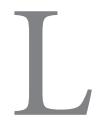
a hipertensión arterial (HTA) es considerada uno de los principales factores de riesgo cardiovascular, aumentando la morbilidad y mortalidad por infarto al miocardio y contribuyendo al desarrollo de enfermedades cardiovasculares, cerebrovasculares y renales ${ }^{1-3}$. Se estima que a nivel mundial, uno de cada cinco adultos tiene presión arterial elevada, es decir, una presión arterial sistólica (PAS) $\geq 140 \mathrm{mmHg}$, y/o una presión arterial diastólica (PAD) $\geq 90 \mathrm{mmHg}^{4}$. En el año 2013 fallecieron 9,4 millones de personas en el mundo por complicaciones asociadas a esta patología ${ }^{5}$. El escenario en Chile es similar, siendo la condición responsable de la mayor carga de en- 
fermedad en el país 6 . La última Encuesta Nacional de Salud 2009-2010 (ENS 2009-2010) evidenció que $26,9 \%$ de la población presentaba esta patología, afectando en mayor proporción a personas con un bajo nivel educacional y aumentando su prevalencia con la edad?

Es conocido que el consumo excesivo de sodio, principalmente a través de la sal común, aumenta el riesgo de HTA y, por ende, el riesgo de enfermedades cardiovasculares ${ }^{2,8}$. Sin embargo, existen otras causas asociadas al desarrollo de esta patología, algunas modificables, como la baja calidad de la dieta, la práctica insuficiente de actividad física, el consumo excesivo de alcohol, el tabaquismo, el exceso de peso y la exposición prolongada al estrés; y otras asociadas a determinantes sociales, como la región de nacimiento, el envejecimiento, la historia familiar, la globalización, los ingresos económicos y el nivel educacional ${ }^{5,9-12}$.

La detección temprana, el tratamiento apropiado y el control de la HTA producen importantes beneficios sanitarios y de índole económico ${ }^{5}$, por lo que identificar precozmente a las personas que podrían presentar mayor o menor riesgo de desarrollarla resulta esencial. Frente a esto, el objetivo de este estudio fue determinar los factores de riesgo asociados al desarrollo de hipertensión arterial en la población chilena.

\section{Materiales y Métodos}

\section{Diseño del estudio}

La muestra seleccionada comprendió 4.901 participantes de la ENS 2009-2010, quienes contaban con información disponible respecto al valor de su presión arterial ${ }^{7}$. La base de datos fue proporcionada por el Departamento de Epidemiología del Ministerio de Salud. La ENS 2009-2010 corresponde a un estudio de prevalencia realizado en hogares en una muestra nacional, probabilística, estratificada y multietápica de 5.412 personas mayores de 15 años con representatividad nacional, regional, y área urbano/rural.

Los participantes firmaron un consentimiento informado y sus resultados les fueron devueltos con recomendaciones y derivación según correspondía ${ }^{7}$. El protocolo de estudio fue aprobado por el Comité de Ética de Investigación de la Escuela de Medicina de la Pontificia Universidad Católica de Chile.

\section{Mediciones antropométricas y metabólicas}

Las mediciones antropométricas fueron realizadas por personal entrenado y han sido descritas en extenso en la ENS 2009-20107. Se clasificó el estado nutricional según índice de masa corporal (IMC) en base a las recomendaciones de la Organización Mundial de la Salud (OMS) ${ }^{13}$. La obesidad central fue definida tras la medición del perímetro de cintura (PC) y en base a los siguientes puntos de corte: $\geq 83 \mathrm{~cm}$ para mujeres $\mathrm{y} \geq 88 \mathrm{~cm}$ para hombres ${ }^{7}$.

Las muestras de sangre, realizadas en ayuno, fueron obtenidas por una enfermera entrenada siguiendo protocolos estandarizados a nivel nacional ${ }^{7}$. Los marcadores metabólicos de glicemia, colesterol (total, HDL, LDL) y triglicéridos fueron medidos con métodos estandarizados y previamente descritos en la ENS junto a sus respectivos puntos de corte ${ }^{7}$. La presión arterial fue medida en tres oportunidades no consecutivas, considerándose una presión arterial elevada a valores de PAS > $140 \mathrm{mmHg}$ y/o PAD > $90 \mathrm{mmHg}^{7}$.

\section{Clasificación de actividad física}

Los niveles de actividad física (AF), el tiempo destinado a las actividades de transporte, y las actividades de intensidad moderada o vigorosa realizada por los participantes, fueron determinados con el cuestionario Global Physical Activity Questionnaire (GPAQ v2) ${ }^{14}$, el cual ha sido validado internacionalmente ${ }^{15} \mathrm{y}$ en población latina ${ }^{16}$. Los niveles de sedentarismo fueron determinados mediante el autoreporte del tiempo destinado a actividades que involucren estar sentado o reclinado durante el tiempo libre o de trabajo. Los niveles de AF total fueron expresadas en METs (Metabolic-energy-equivalents) ${ }^{17}$. Se consideró como punto de corte para inactividad física un gasto energético menor a 600 METs/ $\mathrm{min} / \mathrm{semana}^{14,18}$.

\section{Estimación del índice de dieta saludable}

La estimación del índice de dieta saludable (IDS) fue realizada en base a la metodología reportada por Dussaillant y cols. en el año $2015^{19}$. El IDS se calculó como la suma de los puntajes asignados a cuatro grupos de alimentos: verduras, frutas, cereales integrales y pescados, quedando con un rango posible de puntaje entre 0 a 4 puntos (un 
Tabla 1. Quintiles de consumo de sal según sexo

\begin{tabular}{|ccc|}
\hline Consumo de sal & $\begin{array}{c}\text { Mujeres } \\
\text { (g/día) }\end{array}$ & $\begin{array}{c}\text { Hombres } \\
\text { (g/día) }\end{array}$ \\
\hline Quintil 1 & $<7,4$ & $<7,8$ \\
\hline Quintil 2 & $7,5-8,7$ & $7,8-9,1$ \\
\hline Quintil 3 & $8,8-9,8$ & $9,2-10,3$ \\
\hline Quintil 4 & $9,9-11,3$ & $10,4-11,7$ \\
Quintil 5 & $>11,3$ & $>11,7$ \\
\hline
\end{tabular}

puntaje alto refleja una alimentación saludable, mientras que un puntaje bajo refleja una alimentación no saludable).

El consumo de sal fue estimado mediante la relación sodio/potasio en la orina. En este trabajo el consumo fue estratificado en cinco quintiles: según nivel de consumo y sexo. En la Tabla 1 se visualizan los puntos de corte según quintil y sexo. El consumo de alcohol fue determinado mediante encuesta y se estratificó en bajo, medio y altos niveles de consumo.

Para determinar las variables sobre hábito tabáquico, se consideraron preguntas basadas en el instrumento mínimo de vigilancia de tabaquismo, utilizado por Organización Panamericana de la Salud. Las variables demográficas como edad, sexo, zona geográfica de residencia (urbano/rural), nivel de escolaridad e ingreso económico fueron recolectadas mediante el uso de cuestionarios validados en población nacional ${ }^{7}$. El tiempo promedio destinado a dormir durante la noche fue reportado mediante el uso de cuestionarios, y para efectos de este estudio se clasificó como: bajo $<7 \mathrm{~h}$ al día; normal: 7 a $9 \mathrm{~h}$ al día y alto: $>9 \mathrm{~h}$ por día. El autoreporte de salud y bienestar fue medido a través de cuestionarios y se clasificó como nivel de bienestar bajo, regular y alto.

\section{Análisis estadístico}

Los análisis estadísticos se calcularon utilizando los factores de expansión y ajustando la muestra a la demografía chilena a junio de $2010^{7}$. Para establecer diferencias significativas entre variables de tipo continua, se utilizó la prueba de t-test para muestras independientes y $\chi^{2}$ para variables categóricas.
Para determinar los principales factores asociados al desarrollo de HTA se realizaron análisis de regresión logística. Estos análisis fueron ajustados por edad, sexo, tabaquismo, educación, ingreso económico e IMC. Los datos para estos análisis fueron presentados como Odds ratio (OR) y sus respectivos intervalos de confianza de 95\% IC. Para todos los análisis se utilizó el módulo de análisis de muestras complejas del programa STATA SE v14 y todos los resultados fueron estimados utilizando muestras expandidas según la ENS 2009-2010 ${ }^{7}$. El nivel de significancia fue definido como $\mathrm{p}<0,05$.

\section{Resultados}

En la Tabla 2 se presentan las características generales de la población con presión arterial normal e HTA. Las personas con HTA presentaban una mayor edad, un menor nivel de escolaridad, ingresos económicos más bajos, presentan mayor IMC y PC. En relación a los estilos de vida, personas hipertensas reportaron menores niveles de AF total, de intensidad moderada, vigorosa o de transporte en comparación a personas con presión arterial normal. Sin embargo, el porcentaje de personas fumadoras fue significativamente menor en personas con HTA.

Respecto a los factores asociados al desarrollo de HTA, se pudo determinar que las mujeres presentaron un menor riesgo de desarrollar HTA que los hombres (OR: 0,69 [95\% IC: 0,59 a 0,81], $\mathrm{p}<0,0001)$. Al realizar el análisis por sexo, se determinó que tanto en hombres como en mujeres el riesgo era mayor en personas mayores de 25 años, en quienes presentaban un estado nutricional de sobrepeso, obesidad u obesidad central y en quienes tenían antecedentes familiares de HTA o diabetes mellitus tipo 2 (DMT2) (Figuras $1 \mathrm{y}$ 2). En relación al consumo de sal, el riesgo fue mayor en mujeres de los últimos dos quintiles de mayor consumo de sal (Figura 1), mientras que los hombres, solo el quintil de mayor consumo de sal fue asociado a mayor riesgo de HTA (Figura 2).

No se encontró una asociación significativa entre tabaquismo e HTA en hombres, no obstante, se identificó un menor riesgo en mujeres exfumadoras (OR: 0,77 [95\% IC: 0,60 a 0,99], $\mathrm{p}=0,040$ ) y fumadoras (OR: 0,67 [95\% IC: 0,52 a 0,86$], p=0,002)$. 
Tabla 2. Características de la población según presión arterial

\begin{tabular}{|c|c|c|c|}
\hline & Normal & Hipertenso & Valor $\mathbf{p}$ \\
\hline \multicolumn{4}{|l|}{ Socio-demográficas } \\
\hline Total (n) & 3.314 & 1.587 & - \\
\hline Edad (años) & $39,4 \pm 15,8$ & $61,5 \pm 14,7$ & $<0,0001$ \\
\hline Sexo, Mujeres (\%) & 61,6 & 57,0 & 0,002 \\
\hline Nivel educacional (\%) & & & $<0,0001$ \\
\hline Básica (< 8 años) & 17,5 & 46,0 & \\
\hline Media (< 12 años) & 60,0 & 43,0 & \\
\hline Técnico o Universitaria & 22,5 & 11,0 & \\
\hline Nivel de ingreso (\%) & & & $<0,0001$ \\
\hline Bajo & 52,3 & 63,6 & \\
\hline Medio & 35,4 & 28,2 & \\
\hline Alto & 12,3 & 8,2 & \\
\hline \multicolumn{4}{|l|}{ Antropométricas } \\
\hline $\mathrm{IMC}\left(\mathrm{kg} / \mathrm{m}^{2}\right)$ & $27,1 \pm 5,1$ & $29,5 \pm 5,5$ & $<0,0001$ \\
\hline Estado nutricional (\%) & & & $<0,0001$ \\
\hline $18,5-24,9 \mathrm{~kg} / \mathrm{m}^{2}$ & 35,0 & 17,7 & \\
\hline $25,0-29,9 \mathrm{~kg} / \mathrm{m}^{2}$ & 41,0 & 40,3 & \\
\hline$\geq 30,0 \mathrm{~kg} / \mathrm{m}^{2}$ & 24,0 & 42,0 & \\
\hline Perímetro de cintura $(\mathrm{cm})$ & $94,3 \pm 11,9$ & $101,0 \pm 12,7$ & $<0,0001$ \\
\hline Obesidad central (\%) & 62,3 & 85,2 & $<0,0001$ \\
\hline \multicolumn{4}{|l|}{ Estilos de vida } \\
\hline Actividad física total (MET/h/semana) & $125,8 \pm 147,5$ & $101,9 \pm 136,6$ & $<0,0001$ \\
\hline Actividad física de transporte (min/día) & $51,0 \pm 85,7$ & $44,6 \pm 83,5$ & 0,015 \\
\hline Actividad física moderada (min/día) & $110,0 \pm 153,8$ & $89,7 \pm 137,0$ & $<0,0001$ \\
\hline Actividad física vigorosa (min/día) & $54,3 \pm 125,9$ & $42,1 \pm 112,1$ & 0,001 \\
\hline Prevalencia inactividad física (\%) & 20,9 & 30,9 & \\
\hline Tiempo sedente (h/día) & $3,4 \pm 2,7$ & $3,4 \pm 2,6$ & 0,821 \\
\hline Consumo de frutas y verduras (g/día) & $212,4 \pm 135,6$ & $222,8 \pm 143,3$ & \\
\hline Consumo de sal (g/día) & $9,5 \pm 2,6$ & $10,3 \pm 3,2$ & \\
\hline Consumo de alcohol (g/día) & $54,4 \pm 87,7$ & $50,8 \pm 98,6$ & \\
\hline Tabaquismo (\%) & & & $<0,0001$ \\
\hline Nunca & 37,6 & 48,6 & \\
\hline Ex-fumador & 21,7 & 29,8 & \\
\hline Fumador & 40,7 & 21,6 & \\
\hline \multicolumn{4}{|l|}{ Metabólicas } \\
\hline DMT2 (\%) & 5,7 & 23,2 & $<0,0001$ \\
\hline Síndrome metabólico (\%) & 24,8 & 59,3 & $<0,0001$ \\
\hline Antecedentes familiares de HTA (\%) & 37,7 & 44,8 & $<0,0001$ \\
\hline PAS $(\mathrm{mmHg})$ & $116,4 \pm 11,5$ & $151,8 \pm 21,9$ & $<0,0001$ \\
\hline $\mathrm{PAD}(\mathrm{mmHg})$ & $72,2 \pm 8,4$ & $84,5 \pm 11,9$ & $<0,0001$ \\
\hline
\end{tabular}

Datos presentados como media y su respectiva desviación estándar para variables continuas y como porcentaje para variables categóricas. Valor p representa diferencias significativas entre personas con presión normal e hipertensos, este valor fue estimado con t-test para variables continuas y $\chi^{2}$ para variables categóricas. 


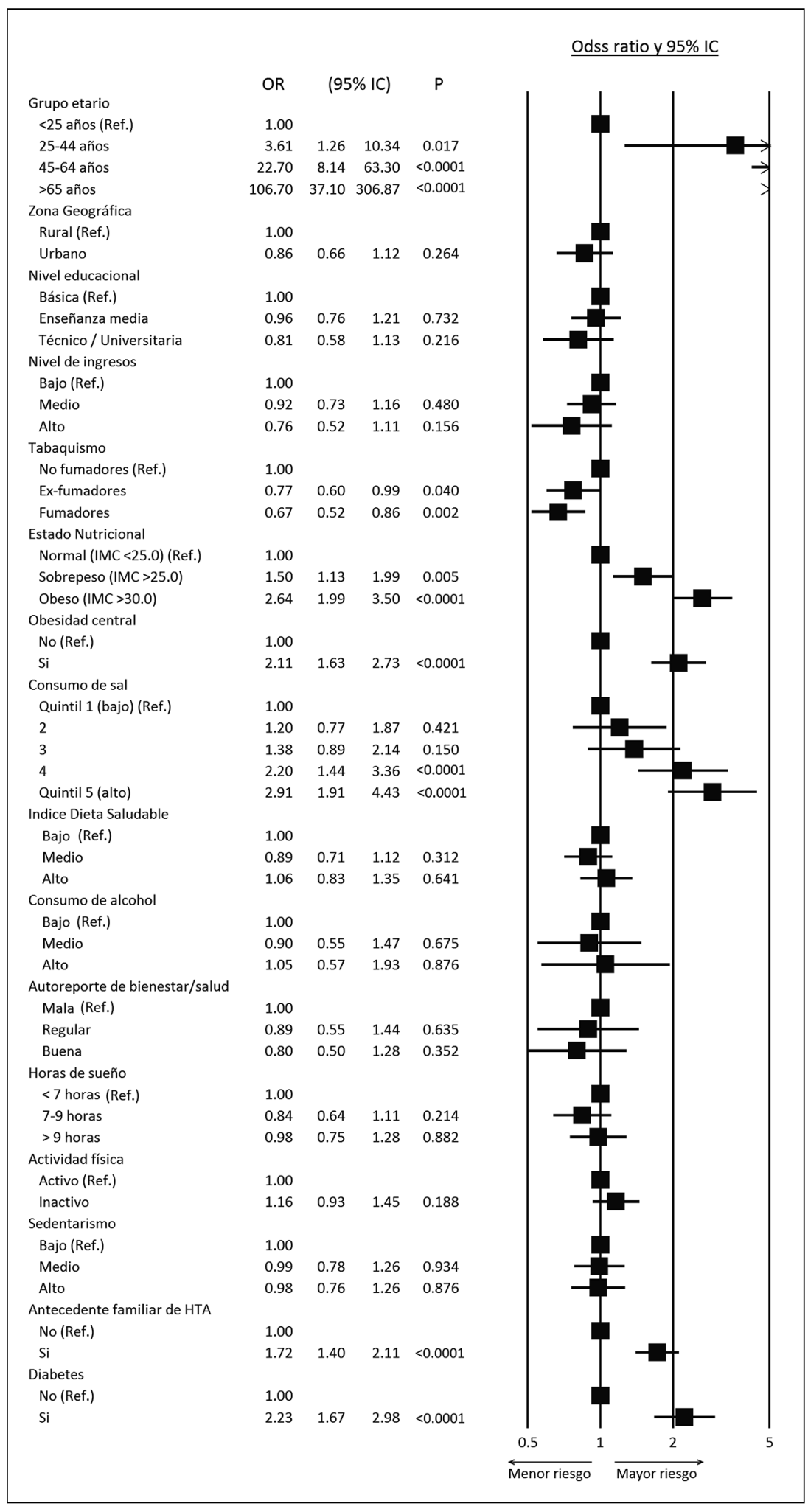

Figura 1. Factores de riesgo asociado a hipertensión arterial en mujeres. Datos presentados como Odds ratio y su respectivo $95 \%$ IC. Los análisis fueron ajustados mutuamente por los factores de riesgo incluidos en la figura (edad, sexo, zona geográfica, educación, ingreso económico, tabaquismo, IMC, consumo de sal, actividad física, sedentarismo, índice de dieta saludable, historia familiar de HTA y DMT2), excepto cuando estos fueron utilizados como factor de riesgo en los análisis. Odds ratio fue estimado mediante regresión logística, y asociaciones significativas fueron consideradas con un valor $p<0,05$. 


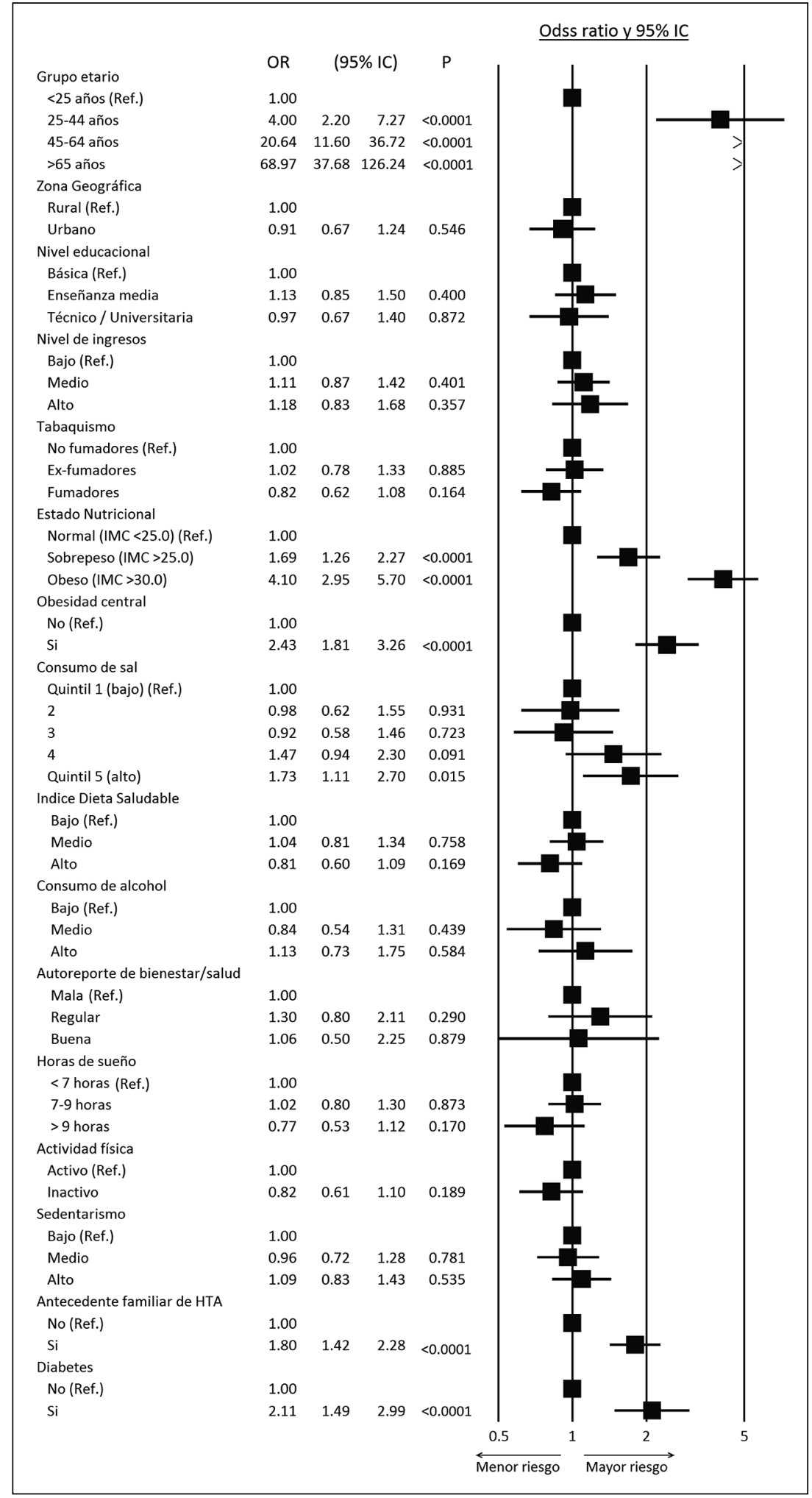

Figura 2. Factores de riesgo asociado a hipertensión arterial en hombres. Datos presentados como Odds ratio y su respectivo 95\% IC. Los análisis fueron ajustados mutuamente por los factores de riesgo incluidos en la figura (edad, sexo, zona geográfica, educación, ingreso económico, tabaquismo, IMC, consumo de sal, actividad física, sedentarismo, índice de dieta saludable, historia familiar de HTA y DMT2), excepto cuando estos fueron utilizados como factor de riesgo en los análisis. Odds ratio fue estimado mediante regresión logística, y asociaciones significativas fueron consideradas con un valor $\mathrm{p}<0,05$. 


\section{Discusión}

Aunque muchos factores de riesgo se asocian con HTA, en este trabajo se logró identificar que el riesgo de presentar hipertensión arterial en Chile es mayor en hombres que en mujeres. Además, este trabajo muestra que, para ambos sexos, el riesgo de presentar HTA aumenta sobre los 25 años de edad, en personas con historia familiar de HTA y con diagnóstico de DMT2. Estos resultados coinciden con lo reportado por otros estudios que señalan que la edad se asocia independientemente con el desarrollo de HTA y que un bajo nivel de educación también influirá en su desarrollo ${ }^{9,11,20}$.

A nivel antropométrico, el exceso de peso es, sin duda, uno de los principales factores de riesgo para el desarrollo de diferentes enfermedades crónicas no transmisibles. Como se evidenció, un IMC mayor $25 \mathrm{~kg} / \mathrm{m}^{2}$ y presentar un PC elevado, son factores independientes para el desarrollo de HTA. Datos similares se han identificado en la literatura, tanto en población occidental ${ }^{21,22}$ como oriental $^{1,11,23}$.

En relación a los estilos de vida, las personas hipertensas reportaron menores niveles de AF y de transporte que aquellas personas con presiones normales $(\mathrm{p}<0,0001)$. A pesar que no se identificó ni en hombres ni en mujeres una asociación estadísticamente significativa entre ser inactivo o sedentario, consumo de alcohol y el desarrollo de HTA, otros trabajos si han establecido que la prevalencia de HTA disminuye en la medida que aumentan los niveles de actividad física ${ }^{24}$ y que el consumo de alcohol en exceso también influye en su desarrollo?.

La disminución del consumo de sal y alimentos procesados es uno de los principales desafíos pendientes, tanto en población general como en población hipertensa. La OMS recomienda un consumo promedio diario de sal no mayor a $5 \mathrm{~g} /$ día en la población ${ }^{25}$ y menor a $3 \mathrm{~g} /$ día en hipertensos, según la etapa de $\mathrm{HTA}^{3}$. El promedio diario reportado por los participantes de este estudio fue de 9,5 g/día y 10,3 g/día en personas con presión arterial normal e hipertensos, respectivamente, cifras muy superiores a las recomendadas y cercanas a los quintiles de riesgo para el desarrollo de HTA en ambos sexos.

Con respecto a la calidad de la dieta, este estudio no identificó diferencias significativas entre el IDS, el consumo de frutas y verduras e
HTA. Sin embargo, es importante enfatizar el rol de la alimentación saludable como medida de promoción y prevención frente a este y todo tipo de patologías crónicas no transmisibles. Si bien es importante la calidad y la cantidad de alimentos consumidos, los horarios y tiempos de comida son parte del equilibrio diario. Aunque esto último no fue investigado en este trabajo, así lo demostró Sic Lee y cols., al evidenciar que las personas que consumen desayuno presentan un menor riesgo de desarrollar $\mathrm{HTA}^{26}$.

A pesar de que numerosos estudios han identificado que el consumo de tabaco es un factor independiente para el desarrollo de HTA $^{11,27,28}$, en este trabajo no se identificó una asociación entre estas variables en hombres, no obstante, en mujeres se identificó que el riesgo disminuía en fumadoras y exfumadoras. Este hallazgo llama la atención, puesto que se han descrito pocos estudios en los cuales existe una disminución en la presión arterial en fumadores ${ }^{29,30}$, como resultado de una disminución del peso corporal por el consumo de tabaco o como un efecto vasodilatador de la cotinina, el principal metabolito de la nicotina ${ }^{31}$. Primatesta y cols. identificaron que fumadoras ligeras (consumo entre 1 a 9 cigarros al día) versus fumadoras pesadas ( $>9$ cigarros al día), presentaban presiones sanguíneas más bajas $^{29}$. En este trabajo no se evaluó la cantidad de cigarros fumados al día, solamente presencia o ausencia de consumo. Se recomienda que futuros estudios puedan incorporar este dato, con el fin de aportar evidencia a esta controversia. A pesar de lo mencionado anteriormente, el tabaquismo debe evitarse en la población general y, por sobre todo, en personas hipertensas, ya que puede aumentar notablemente el riesgo de complicaciones cardiovasculares secundarias y contribuir a la progresión de la insuficiencia renal.

Finalmente, uno de los principales hallazgos de este estudio fue determinar que para ambos sexos el factor que presenta mayor riesgo para el desarrollo de HTA es la edad (> 25 años), seguido de factores de riesgo modificables, como sobrepeso y obesidad, obesidad abdominal, consumo de sal y DMT2. A partir de estos hallazgos y considerando que la HTA representa por sí misma una enfermedad y un factor de riesgo importante para el desarrollo de otras enfermedades cardiovasculares, se debe enfatizar el rol esencial de las estrategias de prevención que se realizan y se deben seguir 
realizando a nivel nacional, recalcando la importancia de disminuir su prevalencia, de generar políticas de salud pública que permitan educar a la población sobre la adopción de estilos de vida saludable, como lo es el disminuir el consumo excesivo de sal y mantener una alimentación balanceada, como también así incorporar la práctica regular de actividad física, factores que juegan un rol muy relevante en combatir la obesidad que es otro factor de riesgo para el desarrollo de HTA.

Agradecimientos: Se agradece de manera especial a todos los participantes de la ENS 2009-10, al equipo profesional de la Escuela de Salud Pública, de la Facultad de Medicina de la Pontificia Universidad Católica de Chile, quienes desarrollaron y aplicaron la Encuesta Nacional de Salud y al Ministerio de Salud del Gobierno de Chile. Todos los autores revisaron críticamente el manuscrito y están de acuerdo con su versión final.

\section{Referencias}

1. Zhang Q, Mahapatra T, Huang F, Tang W, Guo Y, Tang $\mathrm{S}$, et al. Association between Anthropometric Measures and Indicators for Hypertension Control among Kazakh-Chinese Hypertension Patients in Xinjiang, China: Results from a Cross-sectional Study. PLoS One 2017; 12 (1): e0170959.

2. Ohta Y, Kimura Y, Kitaoka C, Sakata T, Abe I, Kawano Y. Blood pressure control status and relationship between salt intake and lifestyle including diet in hypertensive outpatients treated at a general hospital. Clin Exp Hypertens 2017; 39 (1): 29-33.

3. Chobanian AV, Bakris GL, Black HR, Cushman WC, Green LA, Izzo JL, et al. Seventh report of the joint national committee on prevention, detection, evaluation, and treatment of high blood pressure. Hypertension 2003; 42 (6): 1206-52.

4. WHO. Preguntas y respuestas sobre la Hipertensión. World Health Organization. 2015. URL: http://www. who.int/features/qa/82/es/

5. WHO. Información general sobre la HIPERTENSIÓN en el Mundo. World Health Organization. 2013. URL: http://www.who.int/cardiovascular_diseases/publications/global_brief_hypertension/en/

6. Montero J, Mansilla C, Margozzini P. Efecto de la incorporación de registros adicionales a la presión arterial en la Encuesta Nacional de Salud, Chile 2010. Rev Med Chile 2016; 144: 285-90.
7. MINSAL. Encuesta Nacional de Salud 2009-2010. Chile: Ministerio de Salud; 2010. URL: http://web.minsal.cl/ portal/url/item/bcb03d7bc28b64dfe040010165012d23. pdf

8. Mancia G, Oparil S, Whelton PK, McKee M, Dominiczak A, Luft FC, et al. The technical report on sodium intake and cardiovascular disease in low- and middle-income countries by the joint working group of the World Heart Federation, the European Society of Hypertension and the European Public Health Association. Eur Heart J 2017; 38 (10): 712-9.

9. Karmacharya BM, Koju RP, LoGerfo JP, Chan KC, Mokdad AH, Shrestha A, et al. Awareness, treatment and control of hypertension in Nepal: findings from the Dhulikhel Heart Study. Heart Asia 2017; 9 (1): 1-8.

10. Mamudu HM, Paul TK, Wang L, Veeranki SP, Panchal $\mathrm{HB}$, Alamian A, et al. Association Between Multiple Modifiable Risk Factors of Cardiovascular Disease and Hypertension among Asymptomatic Patients in Central Appalachia. South Med J 2017; 110 (2): 90-6.

11. Saeed KM. Burden of Hypertension in the Capital of Afghanistan: A Cross-Sectional Study in Kabul City, 2015. Int J Hypertens 2017; 2017: 3483872.

12. Koch E, Romero T, Romero CX, Akel C, Manriquez L, Paredes $\mathrm{M}$, et al. Impact of education, income and chronic disease risk factors on mortality of adults: does 'a pauper-rich paradox' exist in Latin American societies? Public Health 2010; 124 (1): 39-48.

13. WHO. Obesity: Preventing and managing the Global Epidemic. 2000. URL: http://www.who.int/nutrition/ publications/obesity/WHO_TRS_894/en/

14. WHO. Global Physical Activity Questionnaire: GPAQ version 2.0. World Health Organization; 2009. URL: http://www.who.int/chp/steps/resources/GPAQ_Analysis_Guide.pdf

15. Bull FC, Maslin TS, Armstrong T. Global Physical Activity Questionnaire (GPAQ): Nine Country Reliability and Validity Study. J Phys Act Health 2009; 6 (6): 790804.

16. Hoos T, Espinoza N, Marshall S, Arredondo EM. Validity of the Global Physical Activity Questionnaire (GPAQ) in Adult Latinas. J Phys Act Health 2012; 9 (5): 698-705.

17. Ainsworth BE, Haskell WL, Herrmann SD, Meckes N, Bassett DR Jr, Tudor-Locke C, et al. 2011 Compendium of Physical Activities: A Second Update of Codes and MET Values. Med Sci Sports Exerc 2011; 43 (8): 1575-81.

18. WHO. Global recommendations on physical activity for health. World Health Organization; 2010.

19. Dussaillant C, Echeverría G, Villarroel L, Marín PP, Rigotti A. Unhealthy food intake is linked to higher prevalence of metabolic syndrome in Chilean adult 
population: cross sectional study in 2009-2010 National Health Survey. Nutr Hosp 2015; 32 (5): 2098-104.

20. Rubinstein AL, Irazola VE, Calandrelli M, Chen CS, Gutiérrez L, Lanas F, et al. Prevalence, Awareness, Treatment, and Control of Hypertension in the Southern Cone of Latin America. Am J Hypertens 2016; 29 (12): 1343-52.

21. Suárez JJ, Isakova T, Anderson CA, Boulware LE, Wolf M, Scialla JJ. Food Access, Chronic Kidney Disease, and Hypertension in the U.S. Am J Prev Med 2015; 49 (6): 912-20.

22. Taylor BC, Wilt TJ, Welch HG. Impact of diastolic and systolic blood pressure on mortality: implications for the definition of “normal”. J Gen Intern Med 2011; 26 (7): 685-90.

23. Liao C, Gao W, Cao W, Lv J, Yu C, Wang S, et al. Associations Between Obesity Indicators and Blood Pressure in Chinese Adult Twins. Twin Res Hum Genet 2017; 20 (1): 28-35.

24. Celis-Morales C, Salas C, Álvarez C, Aguilar Farías N, Ramírez Campillos R, Leppe J, et al. Un mayor nivel de actividad física se asocia a una menor prevalencia de factores de riesgo cardiovascular en Chile: resultados de la Encuesta Nacional de Salud 2009-2010. Rev Med Chile 2015; 143: 1435-43.
25. WHO. Reducir el consumo de sal. Nota descriptiva. World Health Organization. 2016.

26. Lee TS, Kim JS, Hwang YJ, Park YC. Habit of Eating Breakfast Is Associated with a Lower Risk of Hypertension. J Lifestyle Med 2016; 6 (2): 64-7.

27. Talukder MA, Johnson WM, Varadharaj S, Lian J, Kearns PN, El-Mahdy MA, et al. Chronic cigarette smoking causes hypertension, increased oxidative stress, impaired NO bioavailability, endothelial dysfunction, and cardiac remodeling in mice. Am J Physiol Heart Circ Physiol 2011; 300 (1): H388-96.

28. Virdis A, Giannarelli C, Neves MF, Taddei S, Ghiadoni L. Cigarette smoking and hypertension. Curr Pharm Des 2010; 16 (23): 2518-25.

29. Primatesta P, Falaschetti E, Gupta S, Marmot MG, Poulter NR. Association between smoking and blood pressure: evidence from the health survey for England. Hypertension 2001; 37 (2): 187-93.

30. Goldbourt U, Medalie JH. Characteristics of smokers, non-smokers and ex-smokers among 10,000 adult males inIsrael. II. Physiologic, biochemical and genetic characteristics. Am J Epidemiol 1977; 105 (1): 75-86.

31. Benowitz NL, Sharp DS. Inverse relation between serum cotinine concentration and blood pressure in cigarette smokers. Circulation 1989; 80 (5): 1309-12. 